Method We performed a cluster randomised trial of community CPR training classes at Nowon District Health Community Centre in Seoul. CPR training classes were randomised into either intervention (instructor's objective real-time feedback based on the QCPR Classroom device or control (conventional, instructor's judgement-based feedback) group. Laerdal QCPR Classroom software is a real-time feedback device, which monitors quality of real-time CPR performances of multiple trainees simultaneously. During each training session, trainees practiced CPR five times. The primary outcome was the total CPR score, which is an overall measure of chest compression quality. Secondary outcomes were individual CPR performance parameters, including average number of compressions, average compression depth, percent adequate depth and percent acceptable release. Generalised linear mixed models were used to analyse the outcome data, accounting for both cluster- (random effect) and individual-(fixed effect) level factors. : A total of 149 training sessions (2613 trainees) were randomised into 70 intervention (1262 trainees) and 79 control (1351 trainees) groups. During the training, trainees in the QCPR feedback group significantly increased overall CPR score performance compared with those in the conventional feedback group (model-based mean $\Delta$ increment from baseline to session 5: 11.2 (95\% CI: 9.2 to 13.2 ) and 8.0 (6.0-9.9), respectively) with statistically significant between group difference $(p=0.02)$. Individual parameters of compression depth and release also showed higher improvement among trainees in QCPR group with marginal significance ( $<<0.08$ for both). Results In this randomised trial, QCPR-Classroom based feedback during the CPR training session resulted in improved overall CPR quality. In this randomised trial, QCPR-Classroom based feedback during the CPR training session resulted in improved overall CPR quality. This suggests beneficial effect of instructor's real-time objective feedback on the quality of layperson's CPR performance.

Conclusion The training equipment used in this study (QCPR Classroom) was supported by the Laerdal Medical, but had no role in the design and conduct of the study; collection, management, analysis, and interpretation of the data.

Conflict of interest None

Funding None

\section{THE INFLUENCE OF AGONAL BREATHING ON CALL- TAKER RECOGNITION OF OUT-OF-HOSPITAL CARDIAC ARREST}

${ }^{1} \mathrm{~S}$ Ball,' ${ }^{1} \mathrm{M}$ Riou, 'S Gallant, ${ }^{1,2} \mathrm{~A}$ Whiteside, ${ }^{1,2,3} \mathrm{P}$ Bailey, ${ }^{1,2,4,5} \mathrm{~J}$ Finn, On behalf of investigators of the NHMRC Partnership Project 'Improving ambulance dispatch to timecritical emergencies'. 'Prehospital, Resuscitation and Emergency Care Research Unit (PRECRU), Curtin University, WA, Australia; ${ }^{2}$ St John Ambulance Western Australia, WA, Australia; ${ }^{3}$ St John of God Hospital Murdoch, WA, Australia; ${ }^{4}$ Department of Epidemiology and Preventive Medicine, Monash University, Victoria, Australia; ${ }^{5}$ Division of Emergency Medicine, The University of Western Australia, WA, Australia

\subsection{6/10.1136/bmjopen-2018-EMS.26}

Aim Call-taker recognition of out-of-hospital cardiac arrest (OHCA) contributes significantly to patient survival, through reduced response time and dispatch-assisted cardiopulmonary resuscitation. A potential barrier to OHCA recognition is agonal breathing, whereby patients are often described as breathing. Our aim was to determine the role of agonal breathing in non-recognition, and delayed recognition, of OHCA.

Method We analysed cases of non-traumatic OHCA where paramedics performed resuscitation, in Perth, Australia in 2014-2015. Calls in this system were processed using the Medical Priority Dispatch System. We excluded cases where the patient was clearly alive during the call. Listening to calls, we categorised answers to the question 'Is s/he breathing?' as 'No', 'Agonal/Ineffective', 'Unsure' or 'Yes'. These data were compared to OHCA recognition, as determined from computer aided dispatch data.

Results Among 902 OHCA cases, 87\% (782/902) were recognised as OHCA by call-takers. In total, 21\% of calls (193/ 902) had answers consistent with agonal breathing (e.g. 'gasping', 'only just'). However, such calls comprised 38\% (46/120) of the cases not recognised as OHCA, and 45\% (112/250) of the cases of delayed recognition (recognised after initial ambulance dispatch). Only $18 \%$ (35/193) of cases with agonal descriptions were recognised as OHCA at initial dispatch.

Conclusion Calls with descriptions of agonal breathing comprise a large proportion of cases that are either not recognised as OHCA or have delayed recognition. Call-taker response to caller descriptions of agonal breathing remains a major opportunity for improved OHCA recognition.

Conflict of interest A Whiteside, P Bailey and J Finn receive salary support from St John Ambulance Western Australia.

Funding Funding for this research was received from an NHMRC (Australian National Health and Medical Research Council) Partnership Project: APP1076949 'Improving ambulance dispatch to time-critical emergencies'.

\section{LAY RESPONDER POST ARREST SUPPORT MODEL: METHODOLOGY \& CONCEPTUAL DESIGN}

${ }^{1} \mathrm{P}$ Snobelen*, ${ }^{2} \mathrm{~J}$ Pellegrino, ${ }^{1} \mathrm{G}$ Nevils, ${ }^{3,4} \mathrm{KN}$ Dainty. ${ }^{1}$ Peel Regional Paramedic Services; ${ }^{2}$ Aultman College; ${ }^{3}$ North York General Hospital; ${ }^{4}$ University of Toronto

10.1136/10.1136/bmjopen-2018-EMS.27

Aim As early as 1993, consideration of the psychological effect of providing CPR on bystanders emerged as an underappreciated concern. One consideration is the ethics of asking people to respond to such emergencies without proper support.

Method The Lay Responder Support Model (LRSM) emerged from the analysis of the data collected after debriefings with 64 lay-responders that participated in an out-of-hospital cardiac arrest. During the first conversations, participants identified the effects of mental trauma, which led to formalise the debriefing process and data collection tools. The program now involves 3 stages: Identifying and Engaging, Debriefing and Follow-up, and Referral for Professional Support.

Results Almost all the cases, lay-responders communicated effects in their daily life, including a wide range of acute physical and/or psychological reactions post event. For some individuals, acute stress reactions caused enough distress to interfere with everyday activities. These findings resulted in the application of Psychological First Aid principles: identifying and facilitating them toward mental health support to promote recovery has wide spread application in traumatic events 